\title{
TEM Study of Epitaxial LSMO-BTO-STO Thin Film Heterostructures
}

Jian-Guo Zheng ${ }^{1}$, Toshihiro Aoki ${ }^{1}$, and Amitesh Paul ${ }^{2}$

1. Irvine Materials Research Institute, University of California-Irvine, Irvine, CA, USA

2. Technische Universität at München, Physik-Department, Lehrstuhl für Neutronenstreuung, Garching, Germany

We have studied microstructure and chemistry of epitaxial $\mathrm{La}_{0.67} \mathrm{Sr}_{0.33} \mathrm{MnO}_{3}$ (LSMO) - $\mathrm{BaTiO}_{3}$ (BTO) thin films grown on $\mathrm{SrTiO}_{3}$ (STO) single crystal substrates by using advanced scanning \& transmission electron microscopy ( $\mathrm{S} / \mathrm{TEM})$. $\mathrm{La}_{0.67} \mathrm{Sr}_{0.33} \mathrm{MnO}_{3}$ (LSMO) is a half metal with attractive ferromagnetic (FM) properties and a Curie temperature $\mathrm{T}_{\mathrm{C}}$ of around $300 \mathrm{~K} . \mathrm{BaTiO}_{3}(\mathrm{BTO})$ shows ferroelectric (FE) effect. A device combining FM and FE thin films may give rise to novel properties because of strong magnetoelectric coupling. The device performance can be influenced by many factors, such as film quality and thickness, interfacial structures, orientation, strain, chemistry and defects. Modern S/TEM techniques may provide such information to understand the film growth and explain device properties.

Figure 1 is the high-angle-annual-dark-field (HAADF) STEM image of a cross-sectional LSMO-BTOSTO heterostructure recorded in the $<010>$ direction of STO. LSMO $(Z=109.73)$ and BTO $(Z=103)$ films show similar $Z$-contrast, but they have much higher intensity than STO $(Z=82)$ in the image. A sharp interface at an atomic scale between BTO and STO can be readily seen. Figure 1 also shows the crystallographic orientation relationships between the materials. The lattice fringes are extended from the substrate to the top layer, without visible interfacial \& threading defects, indicating a high quality epitaxial growth. Figure 2 is an enlargement of interface areas of Figure 1, displaying an atomic arrangement at the LSMO/BTO/STO interfaces. STEM HAADF image clearly indicates that BTO is grown on $\mathrm{TiO}_{2}$ terminated STO surface, where Ti atomic columns have weaker intensity than $\mathrm{Sr}$ atomic columns. The variation of lattice parameters can be determined as well.

X-ray energy dispersive spectroscopy (EDS) was used to reveal the BTO film. Figure 3 shows elemental maps of $\mathrm{Ti}, \mathrm{Ba}, \mathrm{Sr}, \mathrm{La} \mathrm{Mn}$ and $\mathrm{O}$ together with their corresponding Z-contrast image. The Ba map clearly indicates a thin layer of BTO film between the LSMO and STO. Electron energy-loss spectroscopy (EELS) spectrum images (Figure 4) further confirm the existence of BTO thin film with a thickness of three unit cells. There could be intermixing of $\mathrm{Ba}$ and $\mathrm{La} / \mathrm{Sr}$ within a thickness of one unit cell.

To further understand the chemical states of Mn which controls the magnetic properties, EELS nearedge fine structures were studied for both $\mathrm{Mn}$ and $\mathrm{O}$. Figure 5 shows the positions where EELS spectra were collected and Figure 6 the corresponding spectra of oxygen. Detailed analysis of the near-edge fine structures reveals Mn valence state. We also studied the influence of different BTO thicknesses and different STO orientations on the microstructures [1].

References:

[1] The authors would like to acknowledge the use of IMRI facilities. The epitaxial samples were grown by Francis Bern and Binda Chen using PLD technique. 

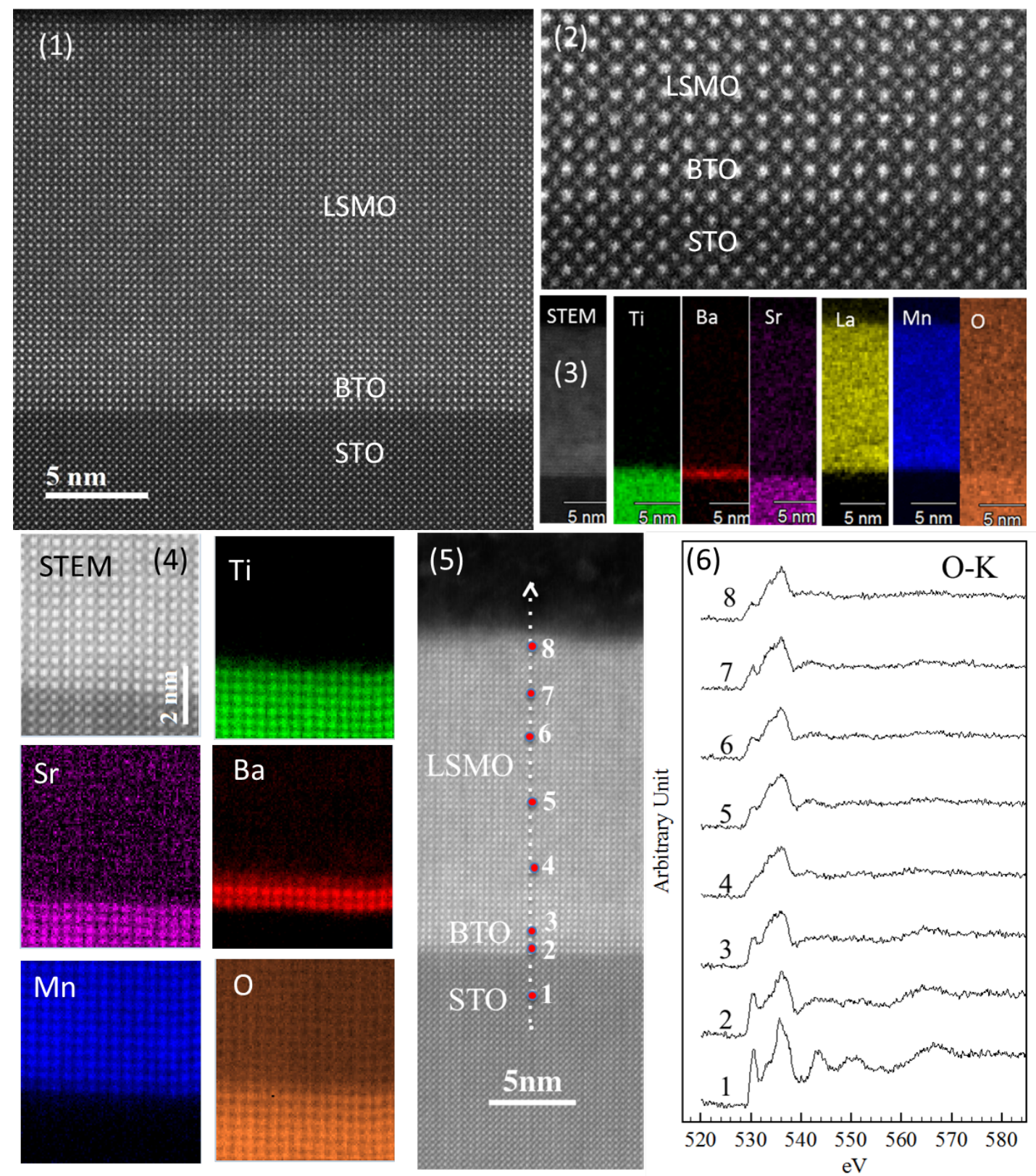

Figure 1. STEM Z-contrast STEM image of epitaxial LSMO-BTO thin films grown on STO (001) substrate.

Figure 2. An enlargement of interface area of Figure 1, showing atomic arrangement at the interfaces. Figure 3. STEM image and EDS elemental maps of Ti, Ba, Sr, La, Mn and $\mathrm{O}$.

Figure 4. STEM image and EELS elemental maps of Ti, Ba, Sr, Mn and $\mathrm{O}$.

Figure 5. STEM images with marked positions where EELS spectra were collected.

Figure 6. EELS spectra of O K-edge. 